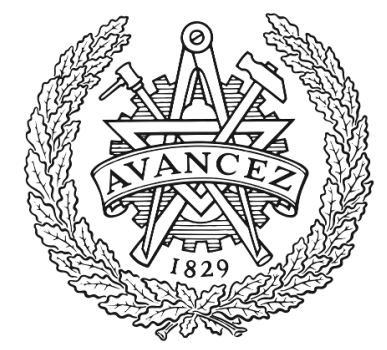

\title{
CHALMERS
}

UNIVERSITY OF TECHNOLOGY

\section{Betatron x-ray radiation from laser-plasma accelerators driven by femtosecond and picosecond laser systems}

Downloaded from: https://research.chalmers.se, 2023-04-26 07:14 UTC

Citation for the original published paper (version of record):

Albert, F., Lemos, N., Shaw, J. et al (2018). Betatron x-ray radiation from laser-plasma accelerators driven by femtosecond and picosecond

laser systems. Physics of Plasmas, 25(5). http://dx.doi.org/10.1063/1.5020997

N.B. When citing this work, cite the original published paper. 


\title{
Betatron x-ray radiation from laser-plasma accelerators driven by femtosecond and picosecond laser systems
}

\author{
F. Albert, ${ }^{1, a)}$ N. Lemos, ${ }^{1,2}$ J. L. Shaw, ${ }^{2, b)}$ P. M. King, ${ }^{1,3}$ B. B. Pollock, ${ }^{1}$ C. Goyon, ${ }^{1}$ \\ W. Schumaker, ${ }^{4}$ A. M. Saunders, ${ }^{5}$ K. A. Marsh, ${ }^{2}$ A. Pak, ${ }^{1}$ J. E. Ralph, ${ }^{1}$ J. L. Martins, ${ }^{6, c)}$ \\ L. D. Amorim, ${ }^{2,6, d)}$ R. W. Falcone,${ }^{5}$ S. H. Glenzer, ${ }^{4}$ J. D. Moody, ${ }^{1}$ and C. Joshi ${ }^{2}$ \\ ${ }^{1}$ Lawrence Livermore National Laboratory, NIF and Photon Sciences, 7000 East Avenue, Livermore, \\ California 94550, USA \\ ${ }^{2}$ Department of Electrical Engineering, University of California, Los Angeles, California 90095, USA \\ ${ }^{3}$ University of Texas Austin, Austin, Texas 78705, USA \\ ${ }^{4}$ SLAC National Accelerator Laboratory, Stanford, California 94309, USA \\ ${ }^{5}$ Lawrence Berkeley National Laboratory and University of California Berkeley, Berkeley, California 94720 , \\ USA \\ ${ }^{6}$ GoLP/Instituto de Plasmas e Fusão Nuclear, Instituto Superior Técnico, Universidade de Lisboa, \\ 1049-001 Lisbon, Portugal
}

(Received 29 December 2017; accepted 10 May 2018; published online 31 May 2018)

\begin{abstract}
A comparative experimental study of betatron x-ray radiation from laser wakefield acceleration in the blowout and self-modulated regimes is presented. Our experiments use picosecond duration laser pulses up to $150 \mathrm{~J}$ (self-modulated regime) and $60 \mathrm{fs}$ duration laser pulses up to $10 \mathrm{~J}$ (blowout regime), for plasmas with electronic densities on the order of $10^{19} \mathrm{~cm}^{-3}$. In the self-modulated regime, where betatron radiation has been very little studied compared to the blowout regime, electrons accelerated in the wake of the laser pulse are subject to both the longitudinal plasma and transverse laser electrical fields. As a result, their motion within the wake is relatively complex; consequently, the experimental and theoretical properties of the x-ray source based on self-modulation differ from the blowout regime of laser wakefield acceleration. In our experimental configuration, electrons accelerated up to about $250 \mathrm{MeV}$ and betatron $\mathrm{x}$-ray spectra with critical energies of about $10-20 \mathrm{keV}$ and photon fluxes between $10^{8}$ and $10^{10}$ photons/eV Sr are reported. Our experiments open the prospect of using betatron $\mathrm{X}$-ray radiation for applications, and the source is competitive with current x-ray backlighting methods on multi-kilojoule laser systems. Published by AIP Publishing. https://doi.org/10.1063/1.5020997
\end{abstract}

\section{INTRODUCTION}

High energy density (HED) plasmas, characterized by very high temperatures $(>10000 \mathrm{~K})$ and pressures ( $>$ Mbar), can be found in planets, stars, or fusion plasmas. Nowadays, these extreme plasma conditions can be generated in laboratories with large scale lasers. Examples include the National Ignition Facility (NIF) and OMEGA in the United States, the Laser MegaJoule (LMJ) in France, and the GEKKO-XII laser in Japan. Most of these HED plasmas are in a nonequilibrium state and are extremely difficult to probe. They are also extremely transient in nature. In laser-driven experiments, an external source of highly penetrating (x-rays) photons is frequently employed as a backlighter probe for HED plasmas. Radiography, absorption spectroscopy, and diffraction are often used to probe the size, density, temperature, or pressure of these highly transient states of matter. At NIF, backlighters are primarily line emission or bremsstrahlung $\mathrm{x}$-ray sources from a laser-solid target interaction. They are used for a number of experiments and platforms such as

\footnotetext{
Note: Paper PI3 2, Bull. Am. Phys. Soc. 62, 262 (2017).

${ }^{a)}$ Invited speaker.

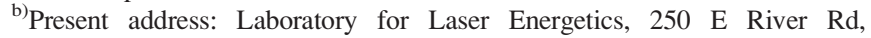
Rochester, New York 14623, USA.

${ }^{c)}$ Present address: Department of Physics, Chalmers University of Technology, Göteborg, Sweden.

${ }^{d)}$ Present address: Department of Physics and Astronomy, Stony Brook University, Stony Brook, NY 11794, USA.
}

convergent ablators (ConA) to measure the ablator density profile $^{1}$ and Target Diffraction In-Situ (TARDIS), where 4 NIF quads (sets of 4 beams) are focused onto a backlighter foil to generate $\mathrm{He}-\alpha \mathrm{x}$-rays. ${ }^{2}$ For Compton radiography, bremsstrahlung $\mathrm{X}$-ray sources are produced by focusing four NIF beams onto a gold nanowire. ${ }^{3}$ More recently, a $13 \mathrm{keV}$ $\mathrm{Kr} \mathrm{K}-\alpha \mathrm{X}$-ray source from gas pipes has been produced with a $3 \%$ conversion efficiency. ${ }^{4}$ However, the current $\mathrm{x}$-ray sources produced using the NIF are isotropic (meaning that most of the photons are not intercepted by the target plasma) and not easily tunable. To produce backlighters with both higher photon yield and energy and short pulse duration, the laser facilities listed above are also equipped with picosecond, petawatt-class systems such as the Advanced Radiographic Capability at LLNL, OMEGA-EP at LLE, the Petawatt Aquitaine Laser (PETAL) at CEA (France), the Orion Laser at AWE (U.K.), and the LFEX laser at the University of Osaka (Japan). Some of these systems have demonstrated x-rays from gold foils, suitable for producing radiographs with $60 \mathrm{keV}$ x-rays. ${ }^{5}$ However, there have been few studies conducted with underdense plasmas to produce $\mathrm{X}$-rays at these facilities. Experiments have been done at OMEGA-EP to understand channeling in underdense targets, requiring the use of plasma plumes produced by hitting a solid $\mathrm{CH}$ target with a longer pulse. ${ }^{6}$ Current $\mathrm{x}$-ray sources are still not highly directional and have large source sizes not achieving the necessary resolution to characterize HED 
plasmas. To address these limitations, we propose to use a laser-wakefield accelerator (LWFA) in these high energy laser facilities, to generate a highly directional, high-energy, small source size and short pulse duration $\mathrm{x}$-ray source.

In our experiments, we are developing an alternate approach for probing HED plasmas that can be implemented on these large-scale facilities. Here, electrons are accelerated via laser-wakefield acceleration (LWFA), where they gain energy in the longitudinal electrical field of the wake produced by an intense but short laser pulse. ${ }^{7}$ Since LWFA was proposed by Tajima and Dawson in $1979,{ }^{8}$ it has achieved many scientific breakthroughs. By definition, a plasma is an ionized medium and therefore can sustain electrical fields, due to space-charge separation, three orders of magnitude higher than that in conventional radiofrequency accelerator structures. A notable application of LWFAs is to generate ultracompact light sources with novel properties, which can have practical applications in industry, security, biology, medicine, condensed matter, and HED science. ${ }^{9,10}$ For example, betatron $\mathrm{X}$-ray radiation is produced when electrons are accelerated at an ultrahigh gradient in a LWFA and then wiggled in the wakefield to emit synchrotron-like radiation. ${ }^{11}$ While betatron $\mathrm{X}$-ray radiation has been mostly studied with laser pulses shorter than $100 \mathrm{fs},{ }^{12}$ most of the facilities capable of producing HED plasmas only have picosecond, kilojoule class lasers. Very little is known about the mechanisms of betatron x-ray radiation in this parameter range, but its implementation on large scale laser facilities could provide a novel probe beam for HED plasmas. This paper presents the first steps towards this realization. We conducted a detailed experimental study of betatron x-ray radiation from a self-modulated laserwakefield accelerator, using picosecond duration laser pulses up to $150 \mathrm{~J}$ and plasmas with electronic densities on the order of $10^{19} \mathrm{~cm}^{-3}$. In this manuscript, we compare the experimental properties of this source with those of more commonly produced betatron x-ray sources with sub 100 fs laser pulses. This paper is organized as follows: Sec. II reviews the physics of electron acceleration mechanisms in the blowout and self-modulated laser wakefield acceleration regimes; Sec. III presents the experimental setup and diagnostics and reviews the difference in x-ray analysis methods for the two regimes; Sec. IV highlights and discusses the main experimental features of betatron $\mathrm{x}$-ray radiation, compares the blowout and self-modulated regimes, and compares the performance of betatron radiation with other sources in the context of HED science. Finally, Sec. V provides a conclusion.

\section{PHYSICS OF BETATRON X-RAY RADIATION IN THE SELF MODULATED REGIME}

\section{A. SMLWA vs. blowout regime}

With sub-100 fs laser pulses, betatron radiation is more commonly produced in the blowout regime of laser-wakefield acceleration. ${ }^{13}$ Here, a laser pulse with length $c \tau$ ( $c$ is the speed of light and $\tau$ the pulse duration) roughly equal to half of a plasma period $\lambda_{p}$ propagates through an underdense plasma (electron density $n_{e}$ of a few $10^{18} \mathrm{~cm}^{-3}$ ) with a normalized vector potential $a_{0}=e A / m c^{2}>2$, which for a $1 \mu \mathrm{m}$ laser corresponds to an intensity of $\sim 4 \times 10^{18} \mathrm{~W} / \mathrm{cm}^{2}$. The laser ponderomotive force plows the electrons away from the strong field regions to form an electron plasma wave with periodicity comparable to the dimensions of the pulse [Fig. 1(a)]. Under certain conditions, electrons can be trapped at the back of the first plasma period and accelerated to $\mathrm{GeV}$-class energies. Meanwhile, electrons trapped slightly off the main laser axis (by a few microns) undergo betatron oscillations as they are accelerated and subsequently emit broadband, synchrotronlike, $\mathrm{keV} \mathrm{x}$-rays. This process is aided by relativistic self-

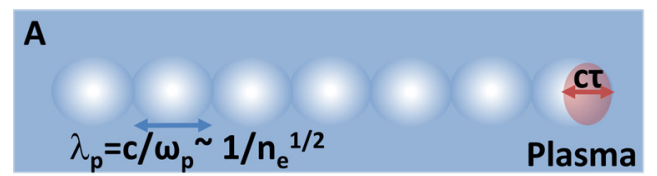

C

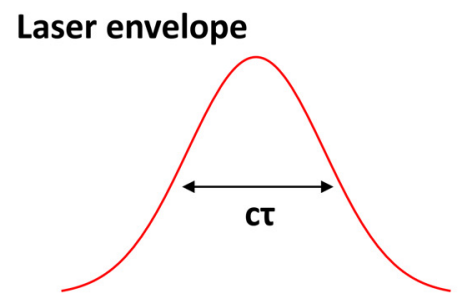

Plasma wave

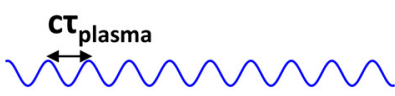

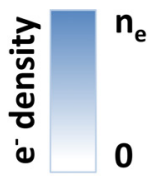

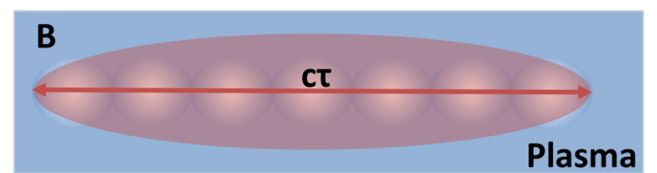

D
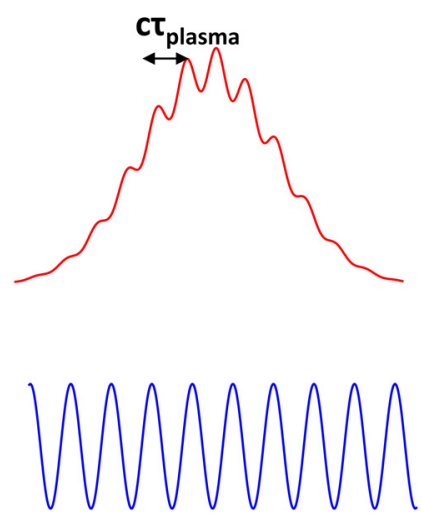

E
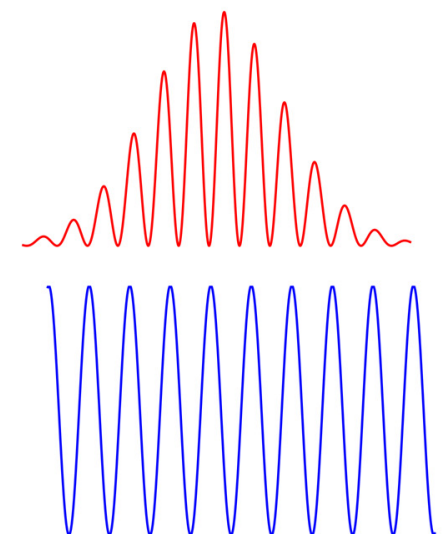

FIG. 1. (a) Blowout regime of LFWA, where the laser pulse length is $\sim$ half a plasma period; (b) self-modulated regime of LWFA, where the laser pulse length overlaps several plasma periods; (c)-(e) evolution of the laser pulse envelope (red) and the plasma wave amplitude (blue) in the self-modulated regime. 
focusing, which occurs if the laser power exceeds the critical power $P_{c}=17\left(\omega_{0} / \omega_{p}\right)^{2} \mathrm{GW}$, where $\omega_{0}$ and $\omega_{p}$ are the laser and plasma frequencies, respectively. For a 30 fs pulse duration, where the matching electron density to be in the blowout regime is a few $10^{18} \mathrm{~cm}^{-3}, P_{c} \sim 2 \mathrm{TW}$. For a 1 ps pulse duration (laser pulse duration of typical lasers in HED science facilities), the matching electron density is about $10^{16} \mathrm{~cm}^{-3}$, and $P_{c} \sim 2 \mathrm{PW}, 3$ orders of magnitude higher than for a $30 \mathrm{fs}$ laser pulse. Since current laser facilities do not have the parameters to achieve this high power, an alternative to produce betatron radiation is to use the self-modulated laser wakefield acceleration (SMLWFA) regime. ${ }^{14}$ Here, the laser pulse overlaps many plasma periods, about 30 for a 1 ps duration pulse and an electron density of $10^{19} \mathrm{~cm}^{-3}$ [Fig. 1(b)]. Thus, the condition to be above $P_{c}$ is achieved only with a few TW, which ps, kilojoule-class laser systems can easily produce. Even if a kilojoule-class picosecond laser system was able to enable experiments in both the blowout and the self modulated regime, recent Particle-In-Cell (PIC) simulations suggest that for conditions relevant to the PETAL laser (kJ, ps), SMLWFA can enhance the betatron X-ray yield by a factor of $10-400^{15}$ compared to the blowout regime. In SMLWFA, the laser pulse propagating through the plasma and interacting with the plasma wave breaks up into trains of shorter pulses, each of them having a length $\lambda_{p}$. This can be explained because the plasma wave has periodic regions of high and low electron density, which causes the laser to successively focus and defocus [Figs. 1(c)-1(e)]. The modulated laser pulse exerts additional forces on the plasma electrons, which leads to the formation of a large amplitude plasma wave, up to the point of wavebreaking, where electrons can be trapped and then accelerated by the plasma wave. Simultaneously to self-modulation, Raman forward scattering, initially forming the electron plasma wave, occurs. ${ }^{16}$ As this process obeys matching conditions for the frequency and wavenumber, $\omega_{0}=\omega_{s} \pm m \omega_{p}$ and $\overrightarrow{k_{0}}=\overrightarrow{k_{s}} \pm m \overrightarrow{k_{p}}$, where $m$ is an integer, the laser spectrum transmitted through the plasma exhibits modulations at the plasma frequency as clear signatures of a self-modulated wakefield. Electrons now can be trapped in several buckets and gain energy from both the longitudinal field of the plasma wave and also directly from the laser field in a process called direct laser acceleration (DLA) ${ }^{17}$ if they overlap the drive laser. ${ }^{18,19}$ Just like in the blowout regime, electrons trapped off axis in SMLWFA undergo betatron oscillations, ${ }^{20}$ which in this case are reinforced by their overlap with the laser pulse. If the laser intensity is increased to above $10^{20} \mathrm{~W} / \mathrm{cm}^{2}$, the SMLWFA structure no longer exists, and electrons, accelerated by DLA alone, ${ }^{21}$ also emit betatron X-rays. ${ }^{22}$

\section{B. Modelling}

The intensity spectrum of betatron $\mathrm{x}$-ray radiation produced in a LWFA is calculated from the direction of observation $\vec{n}$ and from electron trajectories, defined by the particle position $\vec{r}$ and normalized velocity $\vec{\beta}=\vec{v} / c$. The intensity per unit frequency $\omega$ and solid angle $\Omega$ is

$$
\frac{d^{2} I}{d \Omega d \omega}=\frac{e^{2} \omega^{2}}{4 \pi c}\left|\int_{-\infty}^{\infty} \vec{n} \times(\vec{n} \times \vec{\beta}) e^{i \omega\left(t-\frac{\vec{n} \cdot \vec{r}}{c}\right)} d t\right|^{2},
$$

and in the case of on-axis observation, for strong oscillations, the number of photons $N_{x}$ per unit energy approximates to

$$
\frac{d N_{x}}{d E} \propto \frac{1}{E}\left(\frac{E}{E_{c}}\right)^{2} K_{2 / 3}^{2}\left[E / E_{c}\right],
$$

where we define the critical energy $E_{c}$ as the threshold below which half of the x-ray power is radiated.

For ideal conditions in the blowout regime, the electrons do not interact with the laser field (before dephasing) and their motion is governed by the longitudinal and transverse fields of the wake. As a result, electron trajectories can be easily calculated by solving the equation of motion $\frac{d \vec{p}}{d t}=-m \omega_{p}^{2} \frac{\vec{r}}{2}$ $+\alpha \frac{m c \omega_{p}}{e} \overrightarrow{u_{z}}$ with differential equation solving methods, such as a 4th order Runge-Kutta algorithm. The momentum $\vec{p}$ and position $\vec{r}$ are then used as an input to Eq. (1) to calculate the spectrum and beam profile. Although the laser pulse was longer than half a plasma period in our short pulse experiments, this technique worked well to understand the beam profile and spectrum of the betatron source. ${ }^{23}$ The more rigorous method to estimate betatron emission from a laser wakefield accelerator is to calculate electron trajectories with PIC simulations and post process them with the radiation formula. ${ }^{24}$ In the self modulated regime, where the betatron oscillations are also governed by the evolving laser field, it is the correct way to calculate the spectrum. In fact, in our experimental conditions, simulations suggest that the electrons are accelerated by a combination of SMLWFA (at the front of the pulse) and direct laser acceleration (at the back of the pulse). ${ }^{20,25}$ To that effect, DLA has been shown to be present in 2D simulations of LWFA where the laser pulse overlaps the trapped electrons, and DLA is a physical effect (not a numerical artifact) that significantly depends on the longitudinal resolution of the simulation. $^{26}$

\section{EXPERIMENTAL OVERVIEW AND X-RAY ANALYSIS METHODS}

This section presents an overview of the experimental setup used to produce and characterize betatron radiation produced from blowout and self-modulated laser wakefield acceleration. While this paper is not intended to compare the two regimes from the same laser system and to provide an accurate scaling of the source from the blowout to the self modulated regime, it highlights the key features of betatron x-ray radiation from fs-class (blowout regime) and ps-class (self-modulated regime) laser systems at intensities around $10^{18} \mathrm{~W} / \mathrm{cm}^{2}$. For this purpose, a series of experiments were performed on two different lasers at the Jupiter Laser Facility, Lawrence Livermore National Laboratory. The general layout of the experimental setup is shown in Fig. 2. We highlight the main features implemented with each laser system and discuss the key differences in the x-ray analysis methods.

\section{A. Setup}

The Callisto laser typically provided 5-10 J of laser energy for pulse durations of $60 \mathrm{fs}$ and a central wavelength of $\lambda_{0}=800 \mathrm{~nm}$. The laser was focused with an $f / 8$ off-axis 


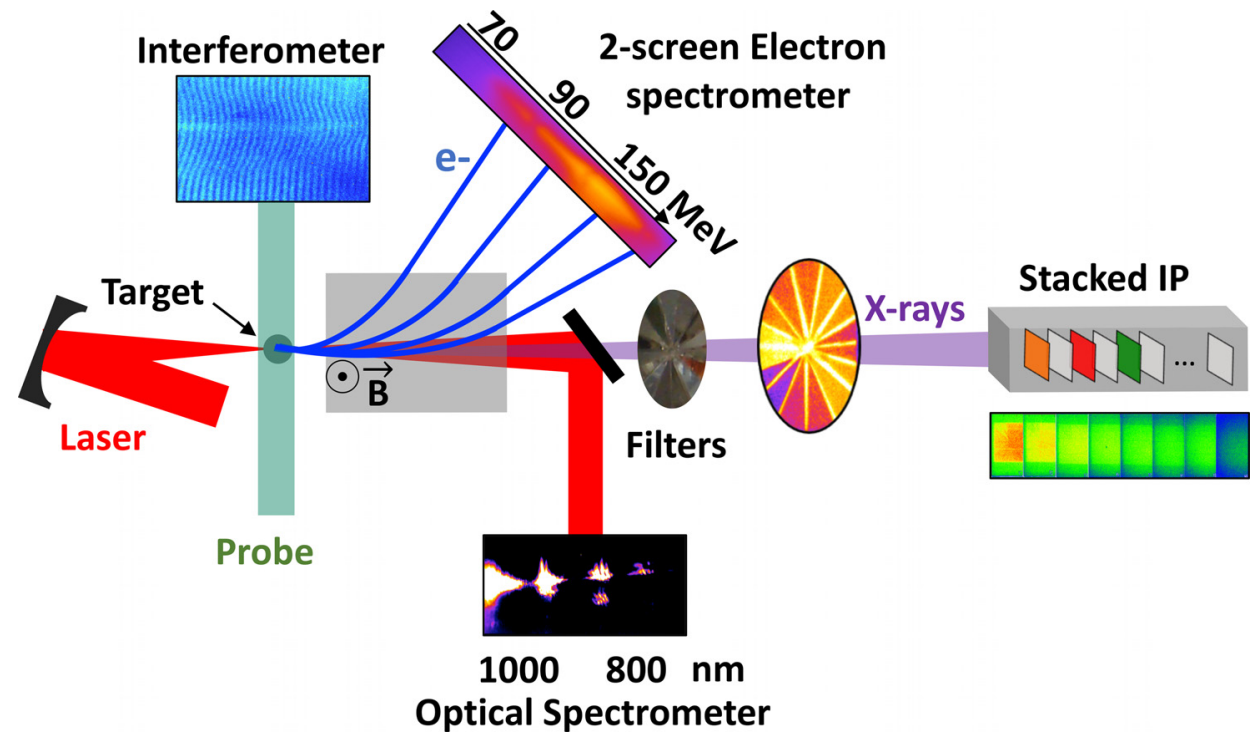

FIG. 2. Experimental setup for the production of betatron $x$-ray radiation in the blowout and self-modulated regimes. The optical spectrometer and $\mathrm{x}$-ray filters were only used for experiments in the self-modulated regime (see text for details).

parabola down to $12 \mu \mathrm{m}$ (full width at half maximum) onto a $10 \mathrm{~mm}$-long gas cell filled with mixtures of helium and nitrogen at electron densities of $\sim 0.5-1 \times 10^{19} \mathrm{~cm}^{-3}$ (the partial pressure of nitrogen could be varied from 0 to $100 \%$ ). For mixed gas $\mathrm{He} / \mathrm{N}_{2}$ shots, the electron injection was dominated by ionization injection ${ }^{27,28}$ and electron energy gain had contributions from both the wakefield and the DLA mechanism. ${ }^{29}$ The Titan laser provides up to $150 \mathrm{~J}$ of laser energy for pulse durations of $0.7_{-0.1}^{+0.3}$ ps and a central wavelength of $\lambda_{0}=1053 \mathrm{~nm}$. It can be focused with an $f / 10$ off-axis parabola down to $29.6 \pm 6.1 \mu \mathrm{m}$ (containing $86 \%$ of the total laser energy) onto a $3 \mathrm{~mm}$ supersonic gas jet, producing electron densities of $\sim 0.1-1.5 \times 10^{19} \mathrm{~cm}^{-3}$. The basic layout of experiments, presented in Fig. 2 for the two laser systems, is very similar, with a few differences, and a more detailed description of the setups is discussed elsewhere. ${ }^{23,25}$ The electron density is measured with interferometry. The electrons, accelerated to a few $100 \mathrm{MeV}$ energies, are deflected onto a 2 -screen electron spectrometer ${ }^{30}$ by a $0.6 \mathrm{~T}, 21.5 \mathrm{~cm}$ long magnet. For the long pulse experiments, the laser spectrum transmitted through the plasma is analyzed with an imaging optical spectrometer. Betatron x-rays, propagating along the main laser axis, can be analyzed with different methods, described in Secs. III B and III C.

\section{B. X-ray analysis: blowout regime}

In the blowout regime, betatron $\mathrm{x}$-ray emission is usually characterized with transmission of x-rays through filters, single photon counting methods, or crystal and grating spectrometers. We recently developed another technique to measure the angular dependence of the betatron x-ray spectrum, ${ }^{23,31}$ well adapted for single shot analysis. In our short pulse experiments, the background noise was sufficiently low and the betatron beam profile well defined that we could rely on a stacked image plate spectrometer to measure the spectrum and beam profile of the source. It consists of image plates arranged in stacks and between which are filters of different materials and thicknesses. With a forward fitting method, where we assume an initial betatron $\mathrm{x}$-ray spectral distribution and calculate what it would produce on the diagnostic, this method allows us to retrieve the source spectrum. For this, we assume an initial spectrum as defined by Eq. (2) and convolve it with the response function of the diagnostic. For each channel, this integrated theoretical signal is compared with the experimental data and reduced through a least squares fitting method, using the spectrum amplitude and critical energy as fitting parameters. An example is shown in Fig. 3.

\section{X-ray analysis: self-modulated regime}

In these experiments, the laser energy $(>100 \mathrm{~J})$ and the high electron beam charge $(\sim 10 \mathrm{nC})$ are responsible for large amounts of background that need to be accounted for. Failure to do this can lead to large overestimations of the betatron source critical energy. Thus, we use the stacked image plates (sensitive to energies above $50 \mathrm{keV}$ in our experimental conditions, where the background dominates) to estimate the

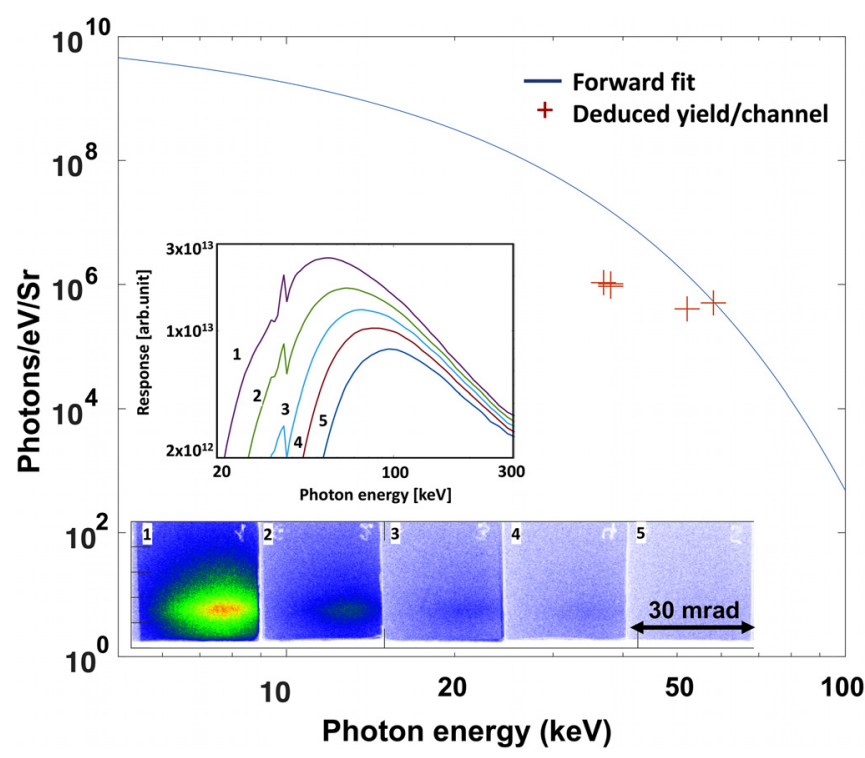

FIG. 3. Data analysis for betatron $\mathrm{x}$-ray radiation in the blowout regime. The bottom image shows the raw data of the stacked image plates. The curve in the inset shows the spectral response of each of the channels to the plotted betatron spectrum. 


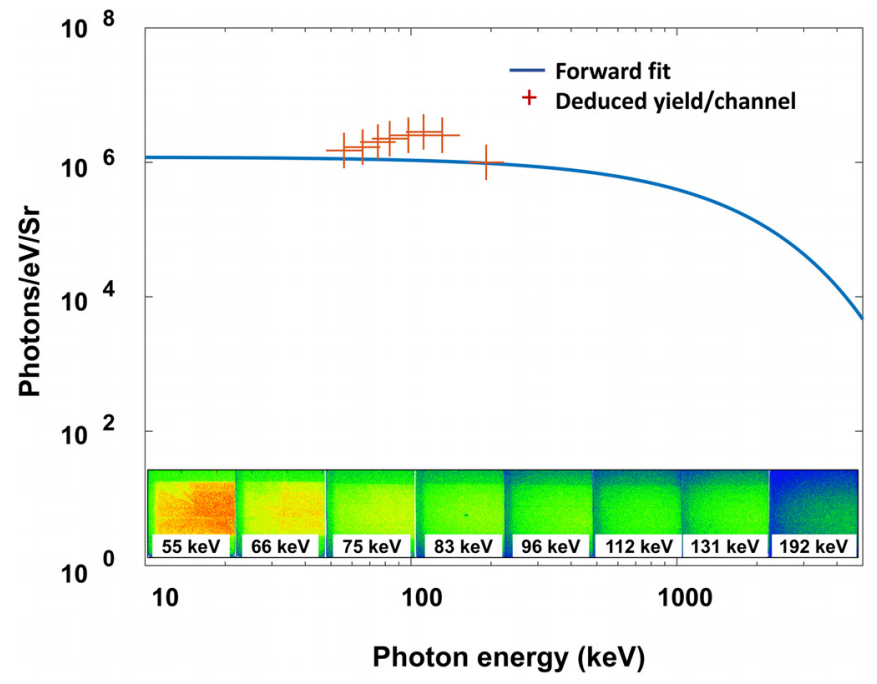

FIG. 4. Data analysis for background noise produced during long pulse experiments. The bottom image shows the raw data of the stacked image plates and the corresponding mean energy of each channel.

background and the thin filters (sensitive to energies below $30 \mathrm{keV}$, where the betatron source dominates) to calculate the betatron source spectrum. Given that the image plate stack is much further from the source $(>1 \mathrm{~m})$ than the magnet where electrons are deflected $(<20 \mathrm{~cm})$, we make the assumption that the background, produced by electrons hitting the chamber walls, is isotropic and has a spectral distribution of the form $A \times \operatorname{Exp}\left[-E / E_{T}\right]$, where $E$ is the photon energy, and the fitting parameters $A$ and $E_{T}$ are the amplitude and the spectrum temperature. This spectral distribution is consistent with the exponentially decaying electron spectra measured during the experiment. We propagate the theoretical spectral distribution through the different materials of the experiment and through the calibrated stacked image plate spectrometer. ${ }^{31,32}$ The total theoretical yield in the plates for $i=1: 8, P_{T, i}$, is calculated and compared to the experimental result $P_{E, i}$ to minimize the residue $\sum_{i}\left(P_{T, i}-P_{E, i}\right)^{2}$ by varying the parameters $E_{T}$ and $A$. An example is shown in Fig. 4, where the deduced spectrum is plotted against the experimental average in each channel. Typical values for $E_{T}$ lie between 0.2 and $0.9 \mathrm{MeV}$. The spectrum obtained from Fig. 4 is then utilized as a background to be subtracted from the thin filter data. The theoretical betatron spectrum [Eq. (2)] is calculated through the different filters of the wheel and integrated to obtain the corresponding signal that it would yield on the image plate. Both the experimental (minus background) and theoretical data are normalized so that the sum of the signals of the filters is equal to 1 . The betatron $\mathrm{x}$-ray critical energy is retrieved through a least squares fitting method by minimizing the number $\sum_{i}\left(D_{i}-T_{i}\right)^{2}$, where $D_{i}$ and $T_{i}$ are the measured and calculated normalized signals for each filter, respectively.

\section{ELECTRON AND X-RAY SOURCE CHARACTERISTICS}

This section presents detailed side-by-side characteristics of the betatron source produced in our experiments with short and long pulse lasers.

With a short pulse laser, in the blowout regime, the electron spectrum often has a peaked structure, and in optimum conditions, the peak can have a relatively narrow energy spread. This can be explained by the fact that for selftrapping, electrons are trapped at the back of the first plasma period (or bubble), and the electron injection is terminated by the beam loading process. The trapped electrons form a short bunch and are accelerated through the same potential until they dephase to generate a narrow energy peak. When we use mixtures of helium and nitrogen, in either the short or the long pulse case, the most tightly bound nitrogen electrons are ionized and trapped near the peak of the laser pulse, directly inside the plasma bubble. Thus, electrons will be continuously injected and favor broader electron spectra. Figure 5(a) shows three electron spectra obtained in similar conditions during our short pulse experiments (which used a combination of self trapping and ionization injection), and these exhibit structures. The electron spectra obtained in the self-modulated regime have a two-temperature distribution of the form $N_{e} \propto e^{-E / T_{1}}+b e^{-E / T_{2}}$ with $13<T_{1}<18 \mathrm{MeV}$ and $20<T_{2}<50 \mathrm{MeV}$ [Fig. 5(b)]. The transmitted laser
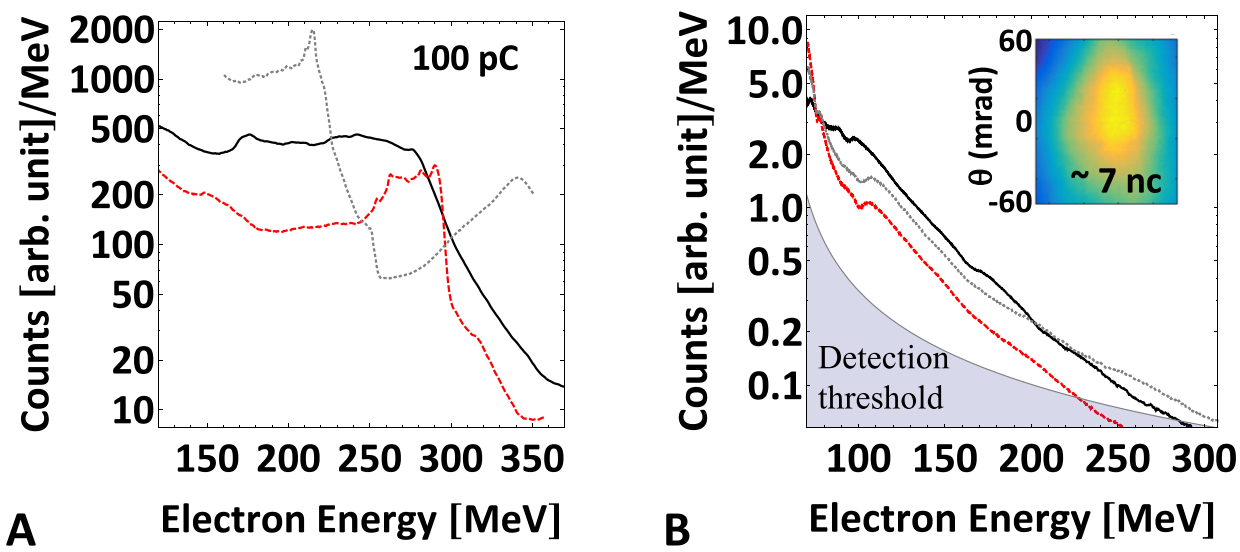

FIG. 5. Electron beam spectra obtained (a) in short pulse experiments (blowout regime) and (b) in long pulse experiments (self-modulated regime). The inset of (b) shows an un-dispersed electron beam. In (a), the laser energy/electron density and gas are $5.3 \mathrm{~J} / 0.6 \times 10^{19} \mathrm{~cm}^{-3}$ and $\mathrm{He}(\mathrm{solid}$ line), $8.5 \mathrm{~J} /$ $0.6 \times 10^{19} \mathrm{~cm}^{-3}$ and $\mathrm{He} / \mathrm{N}_{2} 0.99 / 0.01$ (dashed line), $8.1 \mathrm{~J} / 0.8 \times 10^{19} \mathrm{~cm}^{-3}$ and He (dotted line). In (b), the laser energy/electron density are $150 \mathrm{~J} /$ $0.85 \times 10^{19} \mathrm{~cm}^{-3}$ (solid line), $143 \mathrm{~J} / 0.75 \times 10^{19} \mathrm{~cm}^{-3}$ (dashed line), and $134 \mathrm{~J} / 1.45 \times 10^{19} \mathrm{~cm}^{-3}$ (dotted line). See text for additional details on laser parameters. 


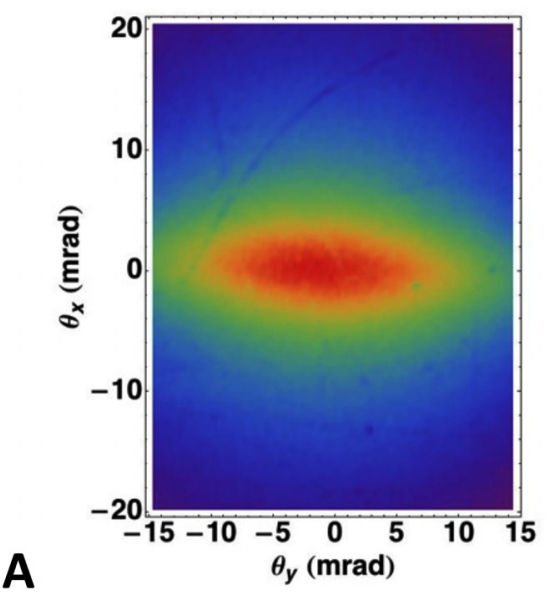

spectra, measured simultaneously, show Raman satellites shifted in frequency by $\pm \omega_{p}$ from the laser frequency, indicative of a self-modulated wakefield. Because of continuous trapping in multiple plasma periods and eventual wavebreaking of the plasma wave, the electron charge (7-10 $\mathrm{nC}$ for electrons above $1 \mathrm{MeV}$ ) is more than 10 times higher in the self-modulated regime.

In our short pulse laser experiments, the betatron $\mathrm{x}$ ray source divergence is typically on the order of 10-50 mrad [Fig. 6(a)]. The beam has, most of the time, an elliptical shape, with the larger axis oriented in the direction of the laser polarization. When they accelerate, the electrons eventually outrun the wake (which travels at the laser group velocity). In our short pulse experiments, the laser pulse (60 fs) was longer than half of the plasma period, and in this case, the accelerating electrons may interact with the laser field before they dephase, which can enhance their oscillation amplitude $r_{0}$ in the direction of the laser polarization axis. The betatron source divergence $\theta$ is defined by $\theta=K \gamma$, where $\gamma$ is the electron relativistic factor, and $K=1.33 \times 10^{10} \sqrt{\gamma n_{e}} r_{0}$ (with $n_{e}$ in $\mathrm{cm}^{-3}$ and $r_{0}$ in $\mu \mathrm{m}$ ). In our long pulse experiments, the betatron beam divergence is typically between 100 and $200 \mathrm{mrad}$. In the self modulated regime, since the electron distribution is Maxwellian, the proportion of low energy electrons is more important, which contributes to an increased divergence. Additionally, the amplitude of the electron oscillations is increased from the beginning because of their overlap with the laser pulse. PIC simulations estimate amplitudes higher than $10 \mu \mathrm{m}$, and the betatron source size, measured with a knife edge around $35 \mu \mathrm{m}\left(1 / e^{2}\right.$ source diameter), confirms this feature.

The X-ray spectra, shown in Fig. 7, and yield are similar in the two regimes, with an $\mathrm{x}$-ray flux on the order of $10^{8}-10^{10}$ photons/eV Sr for our experimental conditions and critical energies between 10 and $40 \mathrm{keV}$, depending on the specific laser and plasma conditions. The number of betatron photons scales linearly not only with the electron charge but also with the wiggler parameter $K$. Hence, the proportion of lower energy electrons in the self modulated regime, which produces fewer photons, is compensated by the higher charge. The selfmodulated regime produced by a ps duration high energy laser is then attractive for single shot HED science experiments on multi kJ-MJ class laser facilities. As explained in Sec. III, the drawback of the self modulated regime (with $\mathrm{kJ}$-class lasers) is to mitigate the noise produced by the electrons in order to detect the $\mathrm{x}$-rays and use them for applications.

In view of developing betatron $\mathrm{x}$-rays as a backlighter for high energy density science, it is useful to compare the performance of this source with other x-ray sources used at high energy density science facilities. Figure 8 presents a number of recent backlighter results. At NIF, line emission, such as $\mathrm{K}-\alpha$ or $\mathrm{He}-\alpha$, is commonly used for radiography or $\mathrm{X}$-ray diffraction studies. ${ }^{33}$ At the OMEGA laser facility, broadband emission from imploding capsules has been used to perform $\mathrm{X}$-ray absorption spectroscopy measurements near the iron $\mathrm{K}$-edge, in order to diagnose shocked compressed iron. ${ }^{34}$ The sandia-Z machine was recently used to probe, with a

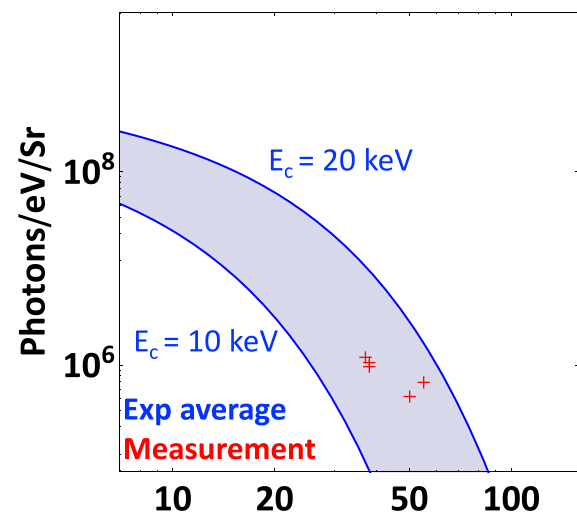

A Photon energy [keV]

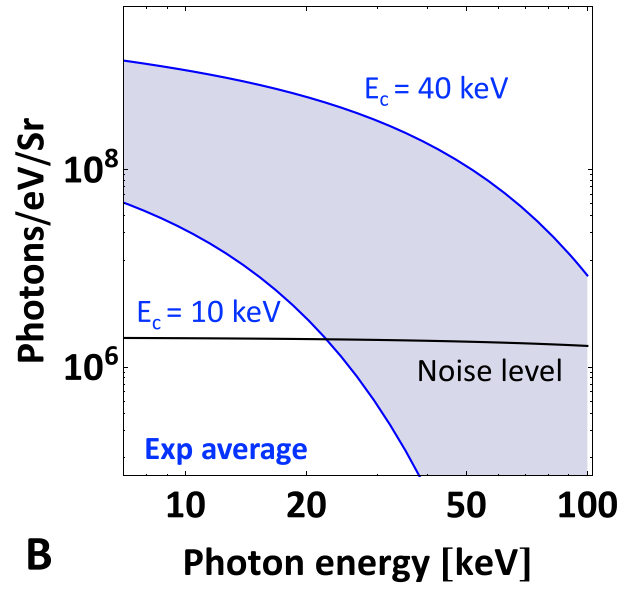

FIG. 7. Average of x-ray spectra obtained (a) in short pulse experiments (blowout regime) and (b) in long pulse experiments (self-modulated regime). See text for additional details on laser parameters and III for additional details on spectrum analysis methods. 


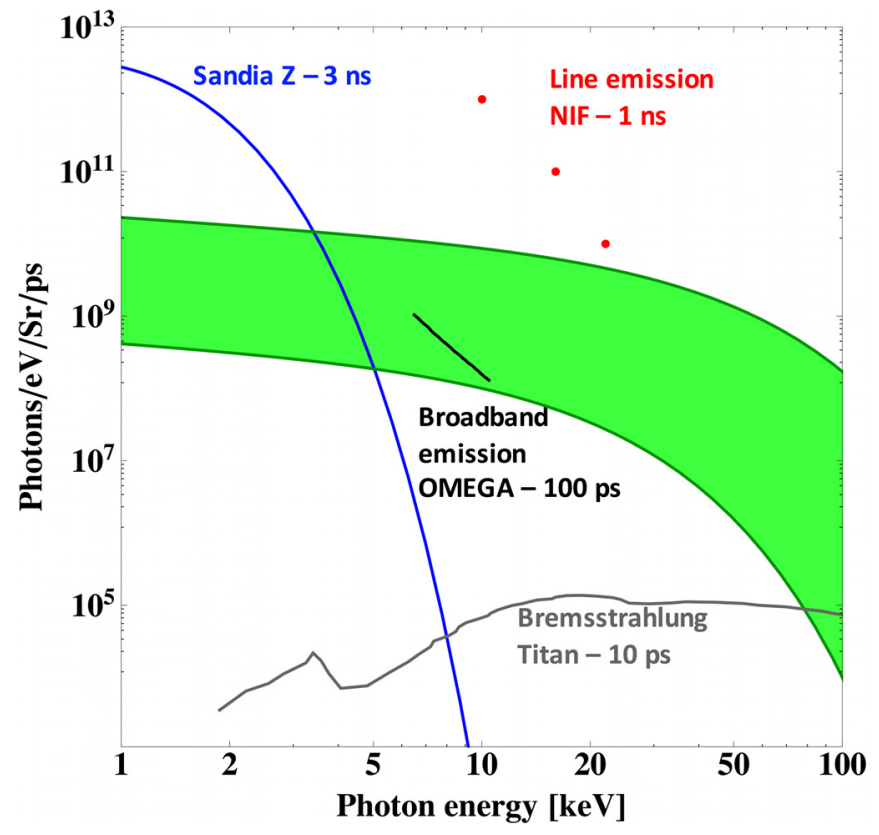

FIG. 8. Overview of x-ray backlighters used at various HED science facilities and comparison with betatron radiation produced by our experiments (green shaded region).

broadband backlighter sufficiently bright to overcome selfemission from the samples, the opacity of iron at solar interior conditions. ${ }^{35}$ Finally, it is important to note that betatron $\mathrm{x}$ ray radiation produced by a mid-scale facility such as Titan at LLNL compares favorably with bremsstrahlung radiation from solid targets at the same laser facility. ${ }^{36}$

\section{CONCLUSION}

In conclusion, we have presented the development of betatron x-ray radiation produced by short (femtosecond) and long (picosecond) pulses. With present laser technologies, the blowout regime, where the laser pulse length is half of a plasma period, is easily produced with short pulse, joule class lasers. However, picosecond laser systems generate laser wakefield acceleration and subsequent betatron x-ray emission in the self-modulated regime. In this case, the electron density is adjusted so that the laser pulse length overlaps tens of plasma periods and leads to wake formation and high-charge electron production via wavebreaking of the wave. The source size and divergence are on the order of ten times bigger in the self modulated regime, due to the larger proportion of low energy electrons and to their higher amplitude oscillations, reinforced by their overlap with the transverse laser field. From our experiments, the x-ray yield produced from a picosecond, $150 \mathrm{~J}$ system is similar to that produced from a $60 \mathrm{fs}, 5 \mathrm{~J}$ system, as it ranges between $10^{8}$ and $10^{10}$ photons/eV Sr. Thus, the photon flux and energy range of the betatron source (Fig. 8) are becoming competitive to other types of $\mathrm{x}$-ray sources being developed at HED science facilities.

This work was performed under the auspices of the U.S. Department of Energy under Contract No. DE-AC5207NA27344 and was supported by the Laboratory Directed
Research and Development (LDRD) Program under tracking codes 13-LW-076 and 16-ERD-024 at LLNL and by the joint NNSA/DOE program Grant No. DE-NA0002950 at UCLA. F.A. acknowledges support from the DOE Office of Science Early Career Research Program under SCW1575-1. R.W.F. acknowledges support from DOE Office of Fusion Energy Sciences under Contract No. DE-AC02-05CH11231. The authors thank R.C. Cauble, S. Andrews, B. Stuart, R. Cross, R. Costa, C. Bruns, C. Cadwalader and S. Maricle for their support of the Titan and Callisto laser systems at the Jupiter Laser Facility. W.S. and S.H.G. acknowledge support from DOE Office of Fusion Energy Sciences under FWP100182.

${ }^{1}$ D. G. Hicks, B. K. Spears, D. G. Braun, R. E. Olson, C. M. Sorce, P. M. Celliers, G. W. Collins, and O. L. Landen, Phys. Plasmas 17, 102703 (2010).

${ }^{2}$ M. F. Ahmed, A. House, R. F. Smith, J. Ayers, Z. S. Lamb, and D. W. Swift, Proc. SPIE 8850, 88500N-1-88500N-8 (2013).

${ }^{3}$ R. Tommasini, S. P. Hatchett, D. S. Hey, C. Iglesias, N. Izumi, J. A. Koch, O. L. Landen, A. J. MacKinnon, C. Sorce, J. A. Delettrez, V. Y. Glebov, T. C. Sangster, and C. Stoeckl, Phys. Plasmas 18, 056309 (2011).

${ }^{4}$ K. B. Fournier, M. J. May, J. D. Colvin, M. A. Barrios, J. R. Patterson, and S. P. Regan, Phys. Rev. E 88, 033104 (2013).

${ }^{5}$ R. Tommasini, C. Bailey, D. K. Bradley, M. Bowers, H. Chen, J. M. D. Nicola, P. D. Nicola, G. Gururangan, G. N. Hall, C. M. Hardy, D. Hargrove, M. Hermann, M. Hohenberger, J. P. Holder, W. Hsing, N. Izumi, D. Kalantar, S. Khan, J. Kroll, O. L. Landen, J. Lawson, D. Martinez, N. Masters, J. R. Nafziger, S. R. Nagel, A. Nikroo, J. Okui, D. Palmer, R. Sigurdsson, S. Vonhof, R. J. Wallace, and T. Zobrist, Phys. Plasmas 24, 053104 (2017).

${ }^{6}$ L. Willingale, P. M. Nilson, A. G. R. Thomas, J. Cobble, R. S. Craxton, A. Maksimchuk, P. A. Norreys, T. C. Sangster, R. H. H. Scott, C. Stoeckl, C. Zulick, and K. Krushelnick, Phys. Rev. Lett. 106, 105002 (2011).

${ }^{7}$ E. Esarey, C. Schroeder, and W. P. Leemans, Rev. Mod. Phys. 81, 1229 (2009).

${ }^{8}$ T. Tajima and J. M. Dawson, Phys. Rev. Lett. 43, 267 (1979).

${ }^{9}$ F. Albert and A. G. R. Thomas, Plasma Phys. Controlled Fusion 58, 103001 (2016).

${ }^{10}$ F. Albert, A. G. R. Thomas, S. P. D. Mangles, S. Banerjee, S. Corde, A. Flacco, M. Litos, D. Neely, J. Vieira, Z. Najmudin, R. Bingham, C. Joshi, and T. Katsouleas, Plasma Phys. Controlled Fusion 56, 084015 (2014).

${ }^{11}$ E. Esarey, B. A. Shadwick, P. Catravas, and W. P. Leemans, Phys. Rev. E 65, 056505 (2002)

${ }^{12}$ S. Corde, K. T. Phuoc, G. Lambert, R. Fitour, V. Malka, and A. Rousse, Rev. Mod. Phys. 85, 1 (2013).

${ }^{13}$ W. Lu, M. Tzoufras, C. Joshi, F. S. Tsung, W. B. Mori, J. Vieira, R. A. Fonseca, and L. O. Silva, Phys. Rev. Spec. Top. Accel. Beams 10, 061301 (2007).

${ }^{14}$ A. Modena, Z. Najmudin, A. E. Dangor, C. E. Clayton, K. A. Marsh, C. Joshi, V. Malka, C. B. Darrow, C. Danson, D. Neely, and F. N. Walsh, Nature 377, 606 (1995).

${ }^{15}$ J. Ferri, X. Davoine, S. Y. Kalmykov, and A. Lifschitz, Phys. Rev. Accel. Beams 19, 101301 (2016).

${ }^{16}$ C. Joshi, T. Tajima, J. M. Dawson, H. A. Baldis, and N. A. Ebrahim, Phys. Rev. Lett. 47, 1285 (1981).

${ }^{17}$ A. Pukhov, Z.-M. Sheng, and J. Meyer-ter-Vehn, Phys. Plasmas 6, 2847 (1999).

${ }^{18}$ C. Gahn, G. D. Tsakiris, A. Pukhov, J. Meyer-ter Vehn, G. Pretzler, P. Thirolf, D. Habs, and K. J. Witte, Phys. Rev. Lett. 83, 4772 (1999).

${ }^{19}$ M. Adachi, E. Miura, S. Kato, K. Koyama, S. ichi Masuda, T. Watanabe, H. Okamoto, A. Ogata, and M. Tanimoto, Jpn. J. Appl. Phys., Part 1 45, 4214 (2006).

${ }^{20}$ N. Lemos, J. L. Martins, F. S. Tsung, J. L. Shaw, K. A. Marsh, F. Albert, B. B. Pollock, and C. Joshi, Plasma Phys. Controlled Fusion 58, 034018 (2016).

${ }^{21}$ S. P. D. Mangles, B. R. Walton, M. Tzoufras, Z. Najmudin, R. J. Clarke, A. E. Dangor, R. G. Evans, S. Fritzler, A. Gopal, C. Hernandez-Gomez, W. B. Mori, W. Rozmus, M. Tatarakis, A. G. R. Thomas, F. S. Tsung, M. S. Wei, and K. Krushelnick, Phys. Rev. Lett. 94, 245001 (2005). 
${ }^{22}$ S. Kneip, S. R. Nagel, C. Bellei, N. Bourgeois, A. E. Dangor, A. Gopal, R. Heathcote, S. P. D. Mangles, J. R. Marquès, A. Maksimchuk, P. M. Nilson, K. T. Phuoc, S. Reed, M. Tzoufras, F. S. Tsung, L. Willingale, W. B. Mori, A. Rousse, K. Krushelnick, and Z. Najmudin, Phys. Rev. Lett. 100, 105006 (2008).

${ }^{23}$ F. Albert, B. Pollock, J. Shaw, K. A. Marsh, J. E. Ralph, Y. H. Chen, D. Alessi, A. Pak, C. E. Clayton, S. H. Glenzer, and C. Joshi, Phys. Rev. Lett. 111, 235004 (2013).

${ }^{24}$ J. Martins, S. Martins, R. Fonseca, and L. Silva, IEEE Trans. Plasma Sci. 39, 2826 (2011).

${ }^{25}$ F. Albert, N. Lemos, J. L. Shaw, B. B. Pollock, C. Goyon, W. Schumaker, A. M. Saunders, K. A. Marsh, A. Pak, J. E. Ralph, J. L. Martins, L. D. Amorim, R. W. Falcone, S. H. Glenzer, J. D. Moody, and C. Joshi, Phys. Rev. Lett. 118, 134801 (2017).

${ }^{26}$ J. L. Shaw, N. Lemos, K. A. Marsh, F. S. Tsung, W. B. Mori, and C. Joshi, Plasma Phys. Controlled Fusion 58, 034008 (2016).

${ }^{27}$ A. Pak, K. A. Marsh, S. F. Martins, W. Lu, W. B. Mori, and C. Joshi, Phys. Rev. Lett. 104, 025003 (2010).

${ }^{28}$ C. McGuffey, A. G. R. Thomas, W. Schumaker, T. Matsuoka, V. Chvykov, F. J. Dollar, G. Kalintchenko, V. Yanovsky, A. Maksimchuk, K. Krushelnick, V. Y. Bychenkov, I. V. Glazyrin, and A. V. Karpeev, Phys. Rev. Lett. 104, 025004 (2010).
${ }^{29}$ J. L. Shaw, N. Lemos, L. D. Amorim, N. Vafaei-Najafabadi, K. A. Marsh, F. S. Tsung, W. B. Mori, and C. Joshi, Phys. Rev. Lett. 118, 064801 (2017).

${ }^{30}$ C. E. Clayton, J. E. Ralph, F. Albert, R. A. Fonseca, S. H. Glenzer, C. Joshi, W. Lu, K. A. Marsh, S. F. Martins, W. B. Mori, A. Pak, F. S. Tsung, B. B. Pollock, J. S. Ross, L. O. Silva, and D. H. Froula, Phys. Rev. Lett. 105, 105003 (2010).

${ }^{31}$ F. Albert, B. B. Pollock, J. L. Shaw, K. A. Marsh, J. E. Ralph, Y.-H. Chen, D. Alessi, A. Pak, C. E. Clayton, S. H. Glenzer, and C. Joshi, Plasma Phys. Controlled Fusion 56, 084016 (2014).

${ }^{32}$ C. D. Chen, J. A. King, M. H. Key, K. U. Akli, F. N. Beg, H. Chen, R. R. Freeman, A. Link, A. J. Mackinnon, A. G. MacPhee, P. K. Patel, M. Porkolab, R. B. Stephens, and L. D. V. Woerkom, Rev. Sc. Instrum. 79, 10E305 (2008).

${ }^{33}$ M. Barrios, K. Fournier, S. Regan, O. Landen, M. May, Y. Opachich, K. Widmann, D. Bradley, and G. Collins, High Energy Density Phys. 9, 626 (2013).

${ }^{34}$ Y. Ping, D. G. Hicks, B. Yaakobi, F. Coppari, J. Eggert, and G. W. Collins, Rev. Sci. Instrum. 84, 123105 (2013).

${ }^{35}$ J. Bailey, Nature 517, 56 (2015).

${ }^{36}$ L. C. Jarrott, A. J. Kemp, L. Divol, D. Mariscal, B. Westover, C. McGuffey, F. N. Beg, M. Suggit, C. Chen, D. Hey, B. Maddox, J. Hawreliak, H.-S. Park, B. Remington, M. S. Wei, and A. MacPhee, Phys. Plasmas 21, 031211 (2014). 